(C) 2019 IEEE. Personal use of this material is permitted. Permission from IEEE must be obtained for all other uses, in any current or future media, including reprinting/republishing this material for advertising or promotional purposes, creating new collective works, for resale or redistribution to servers or lists, or reuse of any copyrighted component of this work in other works. 


\title{
REP18 Atlantic - A Large Scale Exercise Using Unmanned Systems, Field Report
}

\author{
Keila Lima, Paulo Sousa Dias, Maria Costa, José Pinto, Tiago Marques, João Borges Sousa \\ $\ddagger$ LSTS, Dept. of Electrical and Computer Engineering, School of Engineering \\ University of Porto, Portugal \\ Email: \{keila,pdias,mariacosta,zepinto,tiagomarques\}@1sts.pt, jtasso@fe.up.pt \\ Sara Cardigos*, Renato Mendes ${ }^{\dagger \ddagger}$ \\ * Physics Department - University of Aveiro, Portugal \\ $\dagger$ CIIMAR - University of Porto \& CESAM - University of Aveiro \\ Email: $\{$ scardigos,rpsm $\} @$ ua.pt
}

\begin{abstract}
We describe the 2018 edition of the Recognised Environmental Picture exercise (REP18). Every year, the Portuguese Navy hosts an international exercise where challenging and novel operational scenarios are tested in a controlled environment. This is done to achieve a readiness level that allows the use of those developments in future real-world scenarios. The objectives range from military to different scientific purposes, always aiming for the use of innovative though operational technologies. In this paper we document the 2018 objectives and results, as well as innovations that helped resolving the challenges posed by this operational environment enriched by multiple heterogeneous vehicles.
\end{abstract}

Index Terms-Marine operations, Autonomous Underwater Vehicle, Unmanned Aerial Vehicle, Autonomous Vehicle Network

\section{INTRODUCTION}

REP18 Atlantic is a naval exercise organised conjointly by the Underwater Systems and Technology Laboratory from Porto University (LSTS), the Portuguese Navy (PO Navy) and NATO's Centre for Maritime Research and Experimentation (CMRE). REP has been occurring every summer since 2010. This annual event focuses on novel applications of autonomous vehicles for real-world operational scenarios both in defence and science. For this purpose, the organisers bring together world-class players to challenge and see in action both commercial (off-the-shelf) and ongoing research for unmanned systems.

LSTS has been building a fleet of unmanned vehicles, ranging from underwater to surface and aerial that can be used in isolation or coordinated as a team, which has been demonstrated several times at REP exercises. The PO Navy has been operating a series of unmanned vehicles for minecounter measures and rescue operations, together with manned vessels including patrol ships and submarines, which also take part of the exercise. NATO's Centre for Maritime Research and Experimentation (CMRE) from La Spezia, Italy, has been a long time participant in the exercise bringing their expertise on acoustics as well as the NRV Alliance research vessel. Additionally, this year there were participants from the Polish
Navy, US' Naval Undersea Warfare Center (NUWC) and the University of Hawaii.

In REP18, LSTS has operated autonomous vehicles for the following main scenarios:

- Acoustic communications for manned submarines in a distress situation,

- Validation of side-scan sonar, camera and magnetometer for mine detection and identification,

- Scientific data collection for the study of a river estuary and the detection of nonlinear internal solitary waves.

To operate on these challenging scenarios, the LSTS software toolchain for autonomous systems [1] proved to be a significant advantage. Neptus, part of the toolchain, allows seamless operation of multiple autonomous vehicles (of different types) and contains the tools to plan, simulate, execute, monitor, and review the data collected by a team of vehicles.

The operational scenarios for this exercise contain diverse challenges according to their characteristics. Operating autonomous underwater vehicles (AUV) and unmanned aerial vehicles (UAV) have different requirements in terms of operator awareness, data availability and maximum reaction time in case of failures.

All operational scenarios, main and secondary/decoupled ones, involved multidisciplinary teams (see Figure 8), composed by scientists, military personal and operators. In the course of this paper we will describe these scenarios in terms of their planning, requirements, challenges and execution.

\section{TOOLS AND TECHNOLOGIES}

\section{A. Hardware}

For this exercise, LSTS used a set of aerial and underwater autonomous vehicles. Most used in more than one operational scenarios, demonstrating the flexibility and adaptability of the vehicles. Ten different LAUVs have been used by LSTS (a couple more by PO Navy). The LAUV is a man-portable AUV that provides simple and reliable operation. There are three main classes of LAUV vehicles that have been used in REP18: water-column (Xplore class), bottom-mapping (Xtreme class) and experimental (Nemo class). 

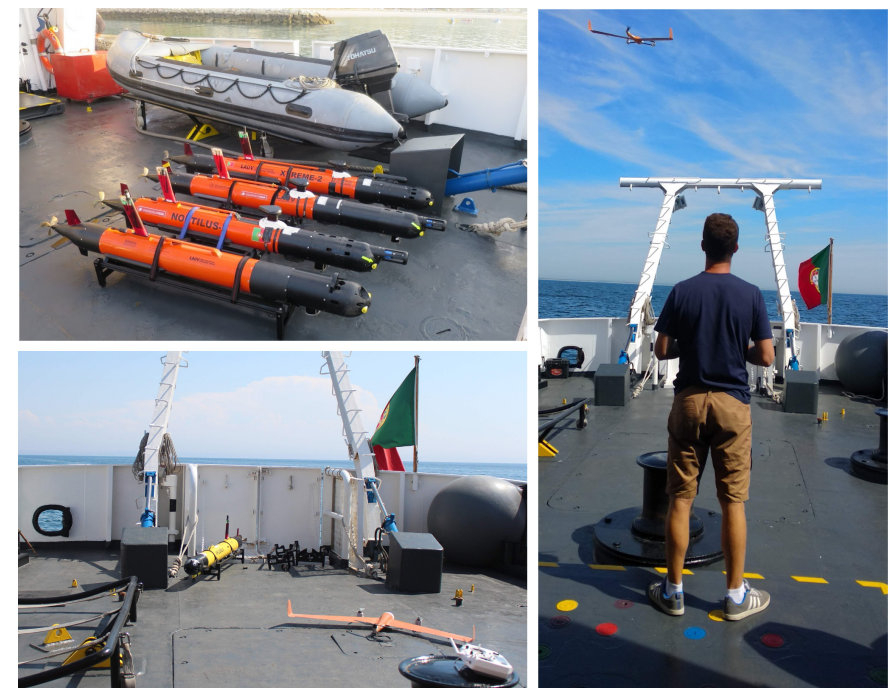

Fig. 1: Some of the harware used by LSTS. Bottom mapping AUVs (top-left), Water column AUV next to VTOL UAV (bottom-left) and VTOL UAV being launched from NRP Auriga (right).

The LAUV Water Column AUVs are designed for open sea missions, being disconnected and far away from the base station for most of the time. For this reason, most communications are done via satellite (Iridium SBD). The localisation on these vehicles is crude, using just MEMS-based IMU, compass and depth for estimating its position underwater. The main application of these vehicles is to measure properties of the water column and, as such, the payloads are typically environmental sensors such as CTD and fluorometers. The battery endurance is also very important and 3 of the 5 Xplore AUVs have 50 hours of endurance at nominal speed $(1 \mathrm{~m} / \mathrm{s})$.

The LAUV Bottom Mapping AUVs are designed to travel close to the bottom in order to survey it. For this reason, they have to cope with obstacles in the bottom as well as being capable of quickly changing their plans in case of incoming traffic (much more common in coastal areas). As such, the AUVs of the Xtreme class use a much more sophisticated localisation system using DVL and tactical grade IMU, which can be further improved using localisation beacons (such as other vehicles) or external USBL modems. To avoid obstacles, the AUVs use a forward-looking echo sounder which detects obstacles at up to 30 meters giving it enough time to stop and ascend to avoid it [2]. In terms of payloads, these vehicles typically carry sidescan sonars, multibeam sonars and (in some cases) magnetometer and optical cameras. For communications the Xtreme AUVs typically use Wi-Fi (close to the base) and GSM (close to the coast), being also possible to use acoustic modem to transfer plans and download information with the vehicles submerged.

Finally, there is another class of AUVs being used that has no specific operational purpose. This results in vehicles that are easy to adapt to perform different experimental scenarios and tests. For instance, the Nemo LAUV is the smallest and lightest of the LSTS fleet but at the same time quite rugged, being possible to launch it from high locations and add several modules like sensing and communication payloads. For REP18, the Nemo LAUV used two modems (Evologics and SeaTrac) and could be used as mobile communications gateway bridging underwater and surface / aerial systems.

LSTS also used the Edge Vertical Take Off And Landing (VTOL) UAV developed by Flightwave Technologies. This type of aircraft is very advantageous in that it can be easily launched and recovered from ships at sea but, at the same time, it can also fly for about 2 hours, giving it an endurance of 100 kms. The Edge also supports different swappable payloads like cameras (infrared, multi-spectral, visible light) and gas sensors. For the REP18 exercise, LSTS tested an infrared camera and long-range telemetry radios, which were recently integrated within the toolchain.

All the vehicles, as well as several Manta gateway devices were operated in different network and payload configurations throughout the exercise. The adaptation to totally different operational concepts in such a small amount of time was only possible by the use of a very capable software layer shared across all devices that we describe next.

\section{B. Software}

The LSTS Toolchain ${ }^{1}$ is an open-source software suite for mixed-initiative control (humans within the planning and control loops) of networked heterogeneous unmanned systems, capable of handling communication challenged environments. The development and evolution of the toolchain has improved greatly over the years with the precious feedback we gather in exercises as REP. In these exercises we have the opportunity to test our vehicles and technologies on real scenarios and also have the chance to interact directly with end users (military personnel, scientists, students, etc.) in an operational context. This exchange of experience and ideas with multidisciplinary teams often results in improvements of the operational concepts and systems being used, either in the implementation of new technologies or in the training/coaching of operators. The Command and Control software developed and used by LSTS materialises much of these interactions in the form of requirements, customisations and visualisations. Neptus [3] is used in three different stages of the operations:

- Mission Planning;

- Operation and monitoring of autonomous/unmanned systems;

- Mission Data Analysis and Review.

This tool allows operators to configure vehicles parameters, plan trajectories, monitor its execution, analyse real-time data or downloaded logs and perform any necessary configuration adjustments to the vehicles. Furthermore, Neptus can also import/export and visualise data in different formats such as S57 charts, KML, GeotiFF, CSV, AIS, etc. These can be overlayed with each other as map layers, which can be useful to correlate different data.

\footnotetext{
${ }^{1}$ LSTS Toolchain source code available at https://github.com/LSTS.
} 
At a lower level, we have the DUNE onboard software running on the different systems, including vehicles and communication support systems. This modular software is divided in several different components that together manage all the hardware, activating different functionalities according to the system where it is running, e.g. AUVs, ASVs, UAVs or even in the Manta communication gateway.

\section{Communication}

Most of the systems use the IMC (Inter-Module Communication) protocol, which is a message-oriented protocol used by all systems to interact with any other nodes in the network [4]. Internally, it is also used for logging proposes and, in DUNE, it is also used to communicate asynchronously between all different modules.

There is also Ripples ${ }^{2}$, a cloud infrastructure used for data dissemination and situation awareness [5]. This tool can be used to follow and control remote assets as it maintains the current status and some collected data of all active systems. Ripples can also be used as an assistant on decision making during operations gathering also remote sensing data, in-situ collected data and AIS information in different layers.

The data between these systems are exchanged by different means: vehicles can use Wi-Fi, acoustics, Iridium satellite, or GSM modules. On the other end the CCUs (Command and Control Units) and ACCUs (Android Command and Control Units) can receive data via Ripples, Manta communication gateway (Wi-Fi or acoustically) and/or via Short Message Service (SMS). It is important to highlight that, despite allowing a wider range of communication, satellite based communications have always an associated cost and can be very limited. The usage of these methods must be efficient and optimised [6].

Acoustic communications was done with the use of modems from Evologics and WHOI's Micromodems. The protocol used in the acoustic is a stripped down version of IMC, to save on bandwidth. In the case of Evologics modems, these can also be used with JANUS (STANAG 4748 [7]), which was the case for some modems during REP18.

\section{Redundant Systems}

For increased safety, there are several alternative methods to locate the vehicles, even when they run out of batteries. Operational procedures acount with these redundancies to mitigate risk.

A GPS tracker system was one of the redundant methods used for the systems' localisation. This technology can have an important role when working in topics such as delay-tolerant networks, where vehicles remain disconnected for long periods of time or in remote and wide operational areas. The trackers can be attached to the vehicles or other assets as, being standalone, they don't require any underlying software or hardware but just a clear view of the sky for normal operation. The locations provided by these devices were integrated into Neptus operation console, allowing operators to be aware of

\footnotetext{
${ }^{2}$ http://ripples.1sts.pt/
}

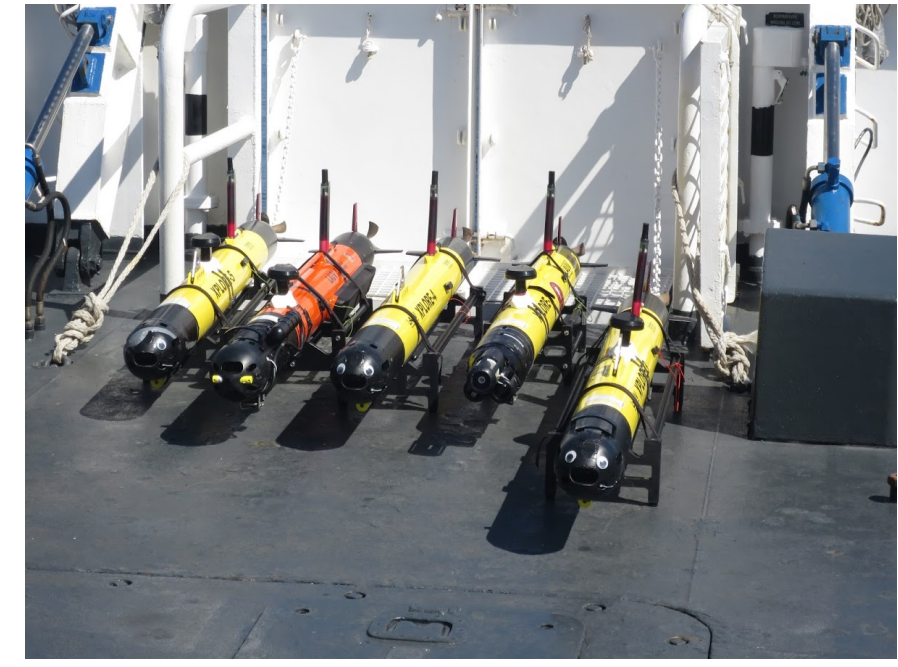

Fig. 2: LAUVs onboard NRP Auriga vessel, during SnOW mission.

the vehicle positions even when disconnected from the IMC network.

An alternative solution to obtain the position of the vehicles even when they're not within wi-fi range is via periodic GSM reports. The periodic reports are sent by the vehicles to a configurable number with information about vehicles status and location. Besides the status report, there is also a set of commands that can be used to control, command and query vehicles status.

With the vehicles submerged, the radios cease to function but there are still more options for monitoring and locating the vehicles. The periodic reports are sent not only via GSM but also over acoustic modem and the same can be used to change the behavior of the AUVs while submerged. Last case scenario, there is a long range standalone acoustic pinger transmitter [2] installed in the front section of the LAUVs. These devices can be tracked using hydrophones tuned in the same frequency as their transmitter and can last from 18 up to 48 months, varying on the configuration.

\section{Digital Acoustic Communications in Sub DISTRESS}

This operation was executed jointly with NATO's CMRE. The scenario consisted in a manned submarine that was submerged and, when entering a distress situation, communicates its state with the surface. We conducted the operation in two stages: first, by using acoustic digital communication based on JANUS (STANAG 4748 [7]) protocol (which allowed the communication of relevant distress information from on-board the submarine as text messages), done by CMRE; During the second stage we processed those distress messages to trigger search and map behaviour on a AUV equipped with an acoustic modem and side-scan sonar.

Traditionally manned submarines distress situations make use of underwater telephone communications. This exercise 
had the objective to make use of digital underwater communications in order to improve the reliability of the data transmitted. This means that, for instance, automatic messages can be triggered in order to periodically send relevant information of the state of the submarine and all of its occupants. With this data being sent digitally, we use it to engage autonomous systems in the search.

We added logic to the AUV in order to listen and respond to distress messages. When a estimated position is read from it, the AUV uses that position to create a search pattern around that point in order to collect side-scan images to access the physical state of the vessel. Additionally to the messages received, ranging the acoustic modem on board the submarine made it also possible to triangulate its position and reduce the search area by reducing the estimated position sent in the distress messages.

This exercise helped to test and evaluate the use of digital acoustic communications and also infer how to use autonomous systems in these distress scenarios.

\section{A. Challenges}

Autonomous underwater vehicles stay longer periods of time disconnected from the operator due to the difficulty of underwater communications that are lower rate and very prone to losses.

\section{B. Results}

The use of digital acoustic communications in a manned submarine in distress situations allows a more reliable passing of information. This information can also be utilised in the search phase. We made use of distance ranging to reduce the position error reported. The use of ranging triangulation helps to better pinpoint the submarine real position which accelerates the search. The used search pattern allowed the successful acquisition of aide-scan sonar images of the submarine. The results from this experiment will drive the REP19 experiment in order to improve the passing of information back to the distressed submarine.

\section{Mine Counter Measures}

In this scenario, the objective was to demonstrate autonomous mine detection using one or more autonomous underwater vehicles. All different participants were given a sheet with the coordinates of different areas and previously sunk targets (without coordinates). The objective was to test different approaches towards the detection and identification of these objects.

LSTS opted to survey an area where multiple diving bottles had been sunk trying to locate them first with sidescan and then identifying all contacts using optical cameras and magnetometer. LSTS tested different sidescan sonars from different vendors (Klein, Edgetech and Imagenex).

\section{A. Results}

All of the sidescans had enough resolution to capture the small air bottles $(17 \times 60 \mathrm{~cm})$. Figure 3 shows some of the

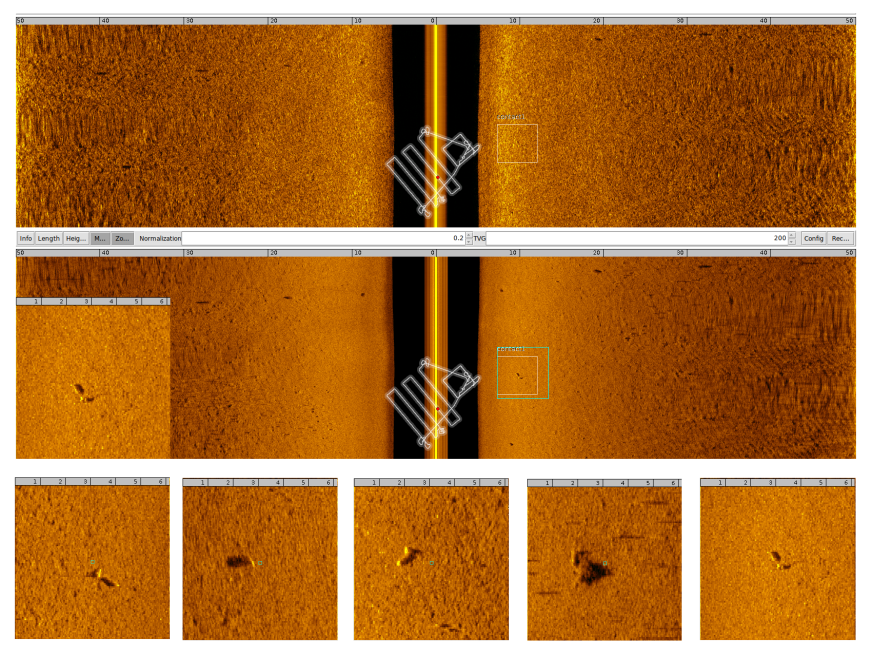

Fig. 3: Partial view of Neptus Mission Review and Analysis application with the contacts detected using a Klein $3500 \mathrm{HF}$ sidescan in the MCM scenario underneath.

contacts that corresponded to the dive bottles (bottom). To mark the contacts, the operators used a sidescan waterfall display that shows the acquired data with the map overlayed, providing tools to measure and export all contacts (top of the image).

After localising the contacts, LSTS did magnetic and optical identification using a bottom-mounted optical camera (custom made by LSTS) and a Self-Compensating Magnetometer (SCM) from Ocean Floor Geophysics. Magnetometers are very sensitive to magnetic disturbances such as those generated by electric motors and, for this reason, they are typically towed behind a vessel or autonomous platform. This poses added complications to the operation and increases the risk of the vehicle becoming entangled in bottom features such as kelp, cables and other fishing equipment. The magnetometer being used by LSTS solves this problem by first measuring the electromagnetic noise generated by the AUV in different conditions and then using this information for compensating the measurements. In practice, the AUV needs to first perform a compensation manoeuvre with similar speed and payload configuration and then perform the survey. As a result, a list of all magnetic anomalies can be obtained (as in figure 4).

The diving bottles could not be identified using just magnetometry probably due to the distance between the LAUV and the bottom $(5 \mathrm{~m})$. The magnetometer data was quite flat and the was no correlation between bottles and anomalies in this case. However, the optical camera was quite capable of capturing the details of the bottles as can be seen in figure 5 .

\section{B. Challenges}

During the tests there some issues with a LAUV being stuck underwater. There were several fish traps in the area which were all connected to each other via long cables. Even though the LAUV was running with no towed instruments it still became entangled in a line underwater. This happened 


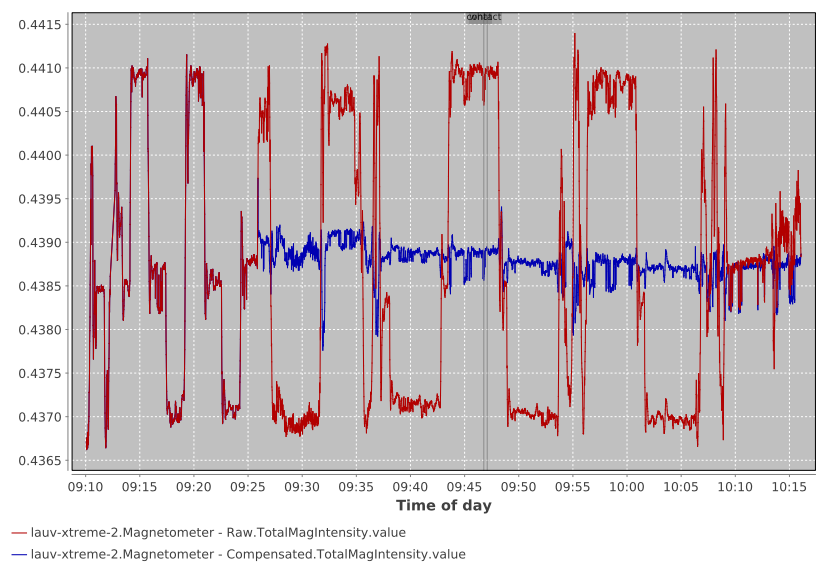

Fig. 4: Raw (red) and compensated (blue) magnetometer data. The compensated data starts diverging from raw only after the compensation maneuver is completed (around 9:25).

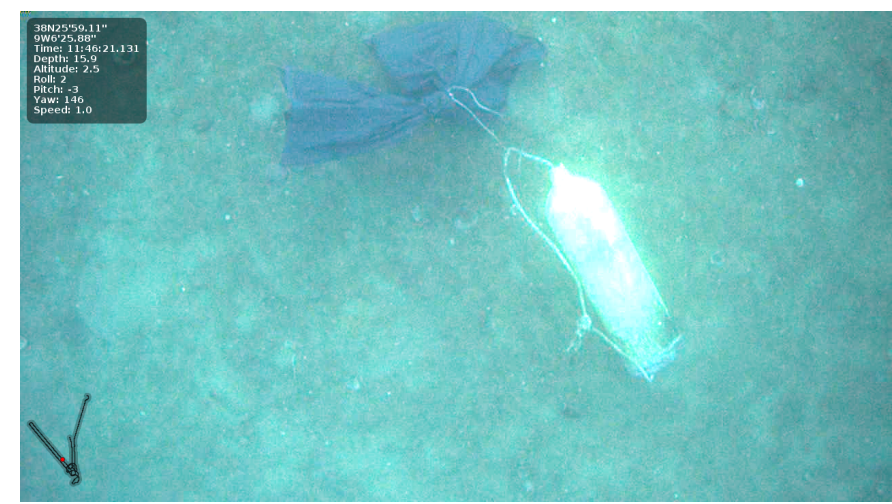

Fig. 5: Dive bottle captured by the onboard camera in LAUVXtreme-2.

once with LAUV when running closer to the bottom (2 $\mathrm{m})$. The magnetometer compensation manoeuvre requires that all payloads that are to be used for the remainder of the mission to be turned on. As a result, a substantial part of the battery is depleted during this time, as the manoeuvre (squares with reversed directions) took around 25 minutes to complete successfully.

\section{SAVEl - SAdo Estuarine OUtFlow Study}

The objective of this scientific scenario was to collect hydrographic data using the AUVs equipped with CTD sensors continuously during 13 hours, to cover the tidal period. The targeted area was the outfall of the Sado estuary, planning to correlate AUVs, UAVs, moorings and remote sensing data. This scenario was successfully executed in a previous exercise edition, in 2017 [8]. The systematic studies of this kind, contributes for a better understanding of the phenomenon being observed, building time-series of data, and helping understand its impact on the stratification of the water column in the region [9]. Even though the objective was similar to last year's, there has been an evolution in the operational concepts. In order to reduce uncertainty of the operators, all commands sent to the vehicles are now acknowledged by the vehicles and the vehicles adapt the execution of their plans in order to better adhere to the plans specified (e.g. by controlling their speed to fulfil the planned ETAs).

\section{A. Challenges}

The operation zone is quite heavy on maritime traffic and comprised an area of about $9 \mathrm{~km}^{2}$, which makes Wi-Fi operations difficult. For this reason, satellite communications were used to operate and monitor the vehicles.

\section{B. Results}

For this mission there were used 4 LAUVs, being 2 of them of the endurance type and operated during the whole time on predefined transects. Another LAUV, equipped with chemical sensors (besides CTD) was used to perform surveys in the same targeted are, while the fourth of them was deployed in a stationary position collecting ADCP data.

Observing the average water temperature distribution along the transect, according to the Figures $6 a$ and $6 \mathrm{~b}$, it is evident a top warmer water layer approximately about $0-5 \mathrm{~m}$ depth in Figure $6 \mathrm{a}$ and approximately about $0-10 \mathrm{~m}$ in Figure $6 \mathrm{~b}$, and a lower water temperature at the $20 \mathrm{~m}$ depth isobath. Regarding vertical stratification, strong mixture is observed in Figure 6a, while in Figure 6b marginal areas present warmer water intrusions. Between the $11^{t h}$ and $19^{t h}$ September it was noticed a water temperature increase (about $0.3^{\circ} \mathrm{C}$ ). Figure $6 \mathrm{~b}$ shows different patterns for the areas under analysis, so these areas were divided in three in order to observe the evolution of each section through time Figure 6c.

\section{SNOW - Study of Nonlinear InTERnal Solitary WAVES}

This exercise's objective was to study nonlinear internal solitary waves in the region of influence of the Sado Estuarine outflow. For this scenario there was a planned combined effort between different data acquisition platforms for an attempt to observe this phenomenon from the surface throughout the water column.

AUVs, UAVs, moorings and remote sensing platforms were also planned to be used during this mission to measure the water velocity, salinity, temperature, turbidity and optical backscatter, correlating the data between different platforms involved.

This mission led to another objective, in the operational side, which was an endurance test to our systems, since this scenario was scheduled to run continuously during 30 hours, in order to cover more than one ebb tidal period.

\section{A. Challenges}

As with the $\mathrm{SaVeL}$ scenario, this operations were also affected by the intense maritime traffic in the operational area, which was also located near the main navigation channel (Portinho da Arrábida). Despite Neptus integrated AIS data 


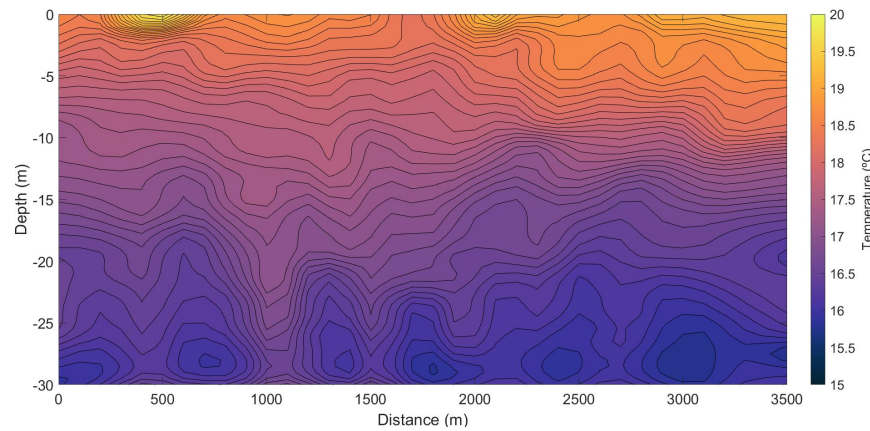

(a) Water temperature average on $11^{\text {th }}$ September.

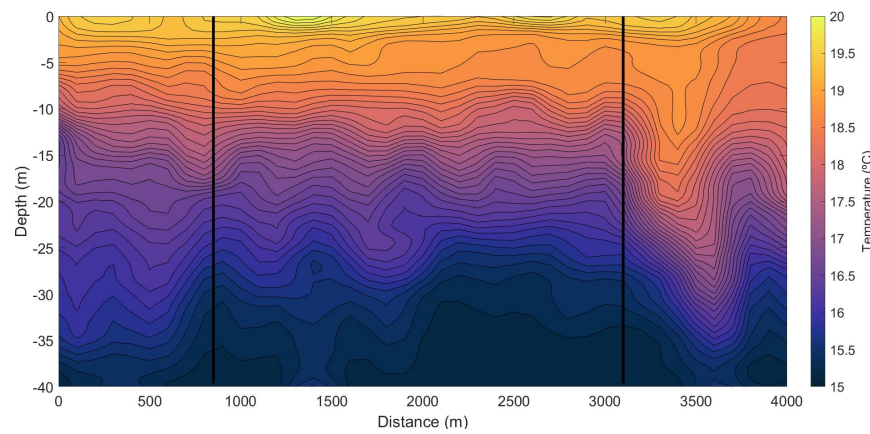

(b) Water temperature average on $19^{t h}$ September.

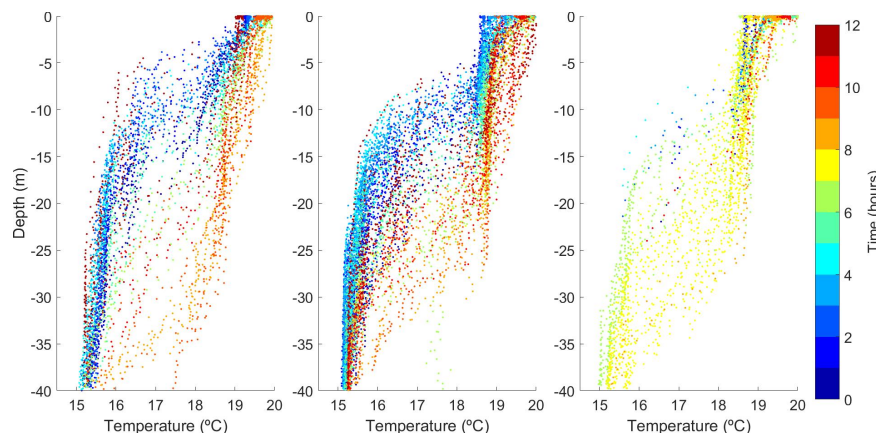

(c) Water Temperature distribution on $19^{t h}$ September.

Fig. 6: SaVel Water Temperature data.

from a receiver installed in the navy vessel, most of the vessels on the area were fishing boats without an identification system and, as a result, the ship's crew collaborated with the operations by providing early warnings whenever they spotted incoming traffic.

\section{B. Results}

The 30 consecutive hours of operations were divided into 4 shifts, where we were able to coordinate the LAUV in situ data collection with satellite passes. UAV flights procedures were also defined, for a better fit into this type of missions.

During the course of the mission, two of the three endurance LAUVs (lauv-xlpore-\{3,4,5\} - depicted in Figure 2) were collecting salinity, temperature and conductivity of the water. Other two were deployed in different timings, in order to maximize the collection of different type of data during the mission, being at the sea over their operating time. The

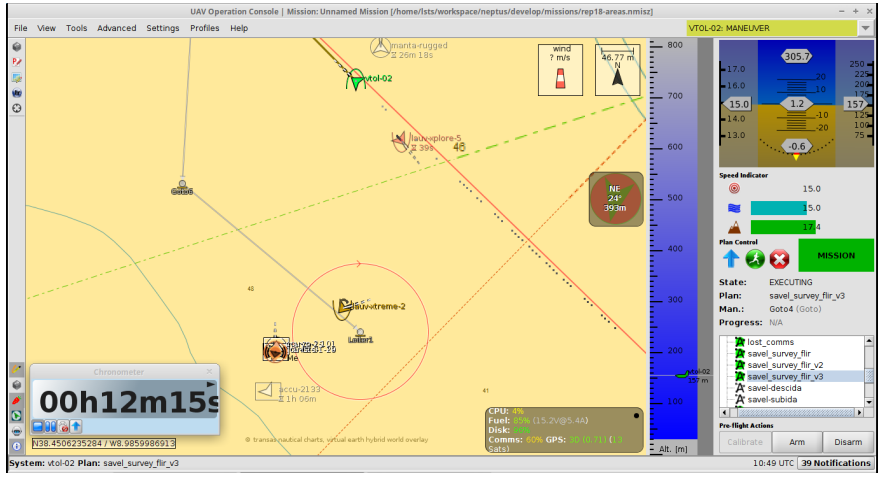

Fig. 7: Neptus console screenshot in AUV and UAV coordinated operation.

first two LAUVs operated coordinately inside a predefined rectangular area $(2 \mathrm{~km}-250 \mathrm{~m})$, repeatedly moving to the opposite corners of the defined area. One of these LAUVs was scheduled to operate during tidal peaks periods (5 times during the mission), being capable of operating for approximately 6 hours. This vehicle was collected periodically to recharge the batteries for the next window of opportunity. The other vehicle (Xtreme class), was used in a stationary position to use its DVL to record water current profiles, mitigating the need for a standalone ADCP sensor which was initially planned for this scenario. The last LAUV, lauv-xplore-2, equipped with more chemical sensors besides CTD, namely turbidity and chlorophyll was the one deployed opportunistically during tide peak periods to perform the same transect over and over again.

\section{Secondary and Decoupled Scenarios}

These scenarios are planned as opportunistic ones, since some exercises can be repeated due to weather conditions or other misfortunes. One example, that has been completed during the 2018 exercise, was the UAV Sea Surface Temperature Collection. Whenever possible, these platforms were planned to flight during low-tide and/or high-tide peaks. This scenario was executed alongside with the other scientific ones, SaVel (see section V) and SnOW (see section VI), being the collected data also relevant to the characterization of the observed phenomenons addressed in each scenario. The efforts in the deployment and coordinated operation of the systems were duplicated in this case. In Figure 7, we can see some of the assets during an UAV coordinated flight with SaVeL surveys performed by one off the LAUVs in the operators console map in Neptus.

This type of scenarios are an opportunity to test minor and/or novel features on the systems, going through all the challenges that are imposed in a real operation scenario.

1) Challenges: Unmanned aerial vehicles keep, for the most time, connection with the operator. Another characteristic of UAVs is that they operate on much higher speeds than the AUVs and so the reaction to abnormal events needs to be quicker. 
2) Results: Besides the UAV sea surface measurements and coordinated operations in different exercises, demonstrated above, LAUV enhancements were also tested. A new vehicle configuration was successfully tested and payload integration on the toolchain is an ongoing process. One of the LAUVs, equipped with a magnetometer, since then, started saving the data internally, in IMC compatible format, with the help of the vehicle's auxiliary CPU. This was the first time that these data could be reviewed directly on the Neptus side, done by filtering the data from the calibration phase of the magnetometer. This feature makes possible a more streamlined and interactive process of data acquisition/review.

\section{CONCLUSIONS}

This yearly exercise greatly contributes for more robust operations in real application scenarios.

We had operations with one or more autonomous underwater vehicles interacting with manned submarines, mine warfare surveys (where we also integrated divers in the operation workflow). We also had coordinated operations between heterogeneous vehicles to perform scientific surveys.

The whole exercise implied the cooperation between heterogeneous systems and multidisciplinary teams forming a complex network. They were successfully carried out due to the usage of a well tested open-source software toolchain, systems and technologies supported by years of field work experience.

Some of these operations occurred simultaneously, in different Navy vessels, which required a flawless coordination of the human resources and an optimal allocation for an effective use of the available assets, in order to fulfil all objectives of each team.

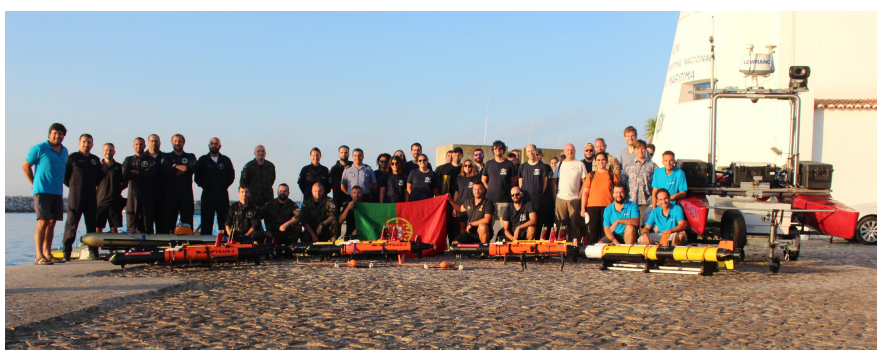

Fig. 8: Group photo taken during REP18 with people and unmanned system from all involved institutions

\section{ACKNOWLEDGMENT}

R.M. benefits from a Post-Doctoral grant (SFRH/BPD/115093/2016) given by the Portuguese Science Foundation (FCT). This work was partially supported by ENDURANCE - Sistema baseado em veículos autónomos para observação oceanográfica de longa duração, supported by Norte Portugal Regional Operational Programme (NORTE 2020), under the PORTUGAL 2020 Partnership Agreement, through the European Regional Development Fund (ERDF); by MARINFO - Integrated Platform for Marine Data Acquisition and Analysis, supported by Norte Portugal
Regional Operational Programme (NORTE 2020), under the PORTUGAL 2020 Partnership Agreement, through the European Regional Development Fund (ERDF) and by the European Union's Horizon 2020 research and innovation programme under grant agreement No 731103.

Finally, the authors are grateful for the extensive support received from the Portuguese Navy.

\section{REFERENCES}

[1] J. Pinto, P. S. Dias, R. Martins, J. Fortuna, E. Marques, and J. Sousa, "The 1sts toolchain for networked vehicle systems," in 2013 MTS/IEEE OCEANS - Bergen, June 2013, pp. 1-9.

[2] L. Madureira, A. Sousa, J. Braga, P. Calado, P. Dias, R. Martins, J. Pinto, and J. Sousa, "The light autonomous underwater vehicle: Evolutions and networking," in 2013 MTS/IEEE OCEANS - Bergen, June 2013, pp. 1-6.

[3] F. Pereira, J. Pinto, J. Sousa, R. Gomes, G. Goncalves, and P. Dias, "Mission planning and specification in the neptus framework," in Proceedings 2006 IEEE International Conference on Robotics and Automation, 2006. ICRA 2006. IEEE, 2006, pp. 3220-3225.

[4] R. Martins, P. S. Dias, E. R. B. Marques, J. Pinto, J. B. Sousa, and F. L. Pereira, "Imc: A communication protocol for networked vehicles and sensors," in OCEANS 2009-EUROPE, May 2009, pp. 1-6.

[5] J. Pinto, P. Sousa Dias, and J. Borges Sousa, "Ripples: a tool for supervision and control of remote assets," in Instrumentation viewpoint, no. 20. SARTI, 2018, p. 51 .

[6] S. Ferreira, J. Pereira, P. Dias, J. Sousa, T. A. Johansen, P. Lourenço, and V. E. Hovstein, "Managing communication challenges in vehicle networks for remote maritime operations," in OCEANS 2017-Aberdeen. IEEE, 2017, pp. 1-7.

[7] R. Petroccia, J. Alves, and G. Zappa, "Janus-based services for operationally relevant underwater applications," IEEE Journal of Oceanic Engineering, vol. 42, no. 4, pp. 994-1006, 2017.

[8] T. Marques, K. Lima, M. Ribeiro, A. S. Ferreira, J. B. Sousa, and R. Mendes, "Characterization of highly dynamic coastal environments, employing teams of heterogeneous vehicles: A holistic case study," in 2018 OCEANS-MTS/IEEE Kobe Techno-Oceans (OTO). IEEE, 2018, pp. $1-8$.

[9] S. Cardigos, R. Mendes, A. S. Ferreira, J. B. de Sousa, and J. M. Dias, "Using lauvs in highly dynamic environments: influence of the tidal estuarine outflow in the thermocline structure," in OCEANS 2018 MTS/IEEE Charleston. IEEE, 2018, pp. 1-6. 